\title{
Evaluation of an Intelligent Utility-Based Strategy for Dynamic Wireless Network Selection
}

\author{
Olga Ormond ${ }^{1}$, Gabriel Miro-Muntean ${ }^{2}$, and John Murphy ${ }^{1}$ \\ ${ }^{1}$ Performance Engineering Laboratory, School of Computer Science and Informatics, \\ University College Dublin (UCD), Dublin, Ireland \\ $\{j$.murphy, olga.ormond\} @ucd. ie \\ ${ }^{2}$ Performance Engineering Laboratory, School of Electronic Engineering, \\ Dublin City University (DCU), Dublin, Ireland \\ munteangleeng. dcu.ie
}

\begin{abstract}
In the case of next generation wireless networks, different users with various multi-homed personal wireless devices will aim to exploit the full potential of the choice of services and applications available over different radio access networks. In their selection of a certain radio access network consumers will consider money and delay preferences for the current application and will rely on intelligent network-selection decision strategies to aid them in their choice. This paper describes the evaluation of an intelligent utility-based strategy for network selection in a multi-access network situation for transfer of large non real-time data files. A number of scenarios are examined which compare the proposed network selection strategy against other possible strategies. Test results show how by using this network selection strategy significant benefits in terms of combined average delay and cost per file transferred as well as transfer efficiency are obtained.
\end{abstract}

Keywords: Heterogeneous Wireless Networks, Multi-homed Radio Terminals, User-centric Network Selection.

\section{Introduction}

Future generations of wireless networks will see the integration and interoperability of a range of current and emerging technologies, and with that an extensive collection of novel and attractive services will be produced by an array of service providers [1]. In order to take advantage of the choice of access networks and the variety of services together with the advances in portable computing, user devices will be built as multihomed devices. The user-centric view for next generation networks is a service oriented heterogeneous wireless network environment (SOHWNE) envisioned as a setting where users and service providers will be free from subscription to any one network operator. They can instead choose the most suitable transport offering from the available network providers for their current mobile terminal and application requirements [2]. End-to-end sessions will be under intelligent terminal control with 
the goal of always connecting to the right access network at the right time to service the current connectivity requirements according to the user preferences.

Section 2 of this paper describes the body of research carried out on the access network selection decision problem. Section 3 then presents the details of our proposed solution to the multi-access decision problem, a Consumer Surplus-based network selection strategy for non real-time data. Using predicted rates for the various available networks we find an intelligent decision strategy for maximising the users' cost savings, while meeting data delay constraints. The dynamic access network selection scenario for large files considered in this paper is presented in section 4 . Section 5 outlines the testing scenarios in which the proposed strategy is compared against an Always Cheapest (AC) strategy, a Network Centric (NC) strategy, a random strategy, and a strategy from the literature. The paper ends in section 7 with conclusions and future work directions.

\subsection{Motivation}

A multi-mode terminal in a heterogeneous Radio Access Network (RAN) environment may be exposed to any combination of RANs (such as GPRS, UMTS, WLAN, WIMAX, Bluetooth, Satellite) at any time. Available bit rates on different technologies affect the types of service and the range of service quality levels that can be offered to users. The range of innovative data applications increases as the range of data rates improves. The intense competition in the marketplace will mean that both access and service providers are eager to survive and thrive by satisfying user demand for 24 hour ubiquitous access to online services. All providers are under pressure to supply the demand for user perceived quality services at the right price, meeting elevated bandwidth demands and supplying flexible services. These services need to be dynamically adaptable to the current context, such as the user terminal capabilities, preferences, and available access network characteristics.

The user-centric view is often referred to in the literature as the Always Best Connected $(\mathrm{ABC})$ concept. Hotspots in airports, coffee shops and other public places already encourage mobile users with a dual terminal in an area of overlapping coverage to avail of the choice of their subscribed operator or a possibly cheaper public hotspot. Their choice will, of course, depend on a number of parameters based on application characteristics, monetary cost, user perceived quality of service, security and other networking concerns. As WLANs become more advanced, with established and secure billing systems they will increase in popularity. Users with private wireless home or office networks may also switch between these networks and the available public networks. However, the user cannot be expected to monitor these RANs, to assess the benefits from using these networks and switch manually to the RAN that best matches user expectations in terms of delay and cost. Therefore an intelligent and automatic solution to network selection is required.

\section{Related Works}

Much of the work in RAN selection policy design investigates the access network selection problem as part of the seamless handover venture. However, access 
selection is also relevant to the initial network selection decision for any user with a choice in available RANs.

Providing a positive user experience is crucial to the success of next generation networks, it is therefore important to take the user preferences into account in the access selection decision. Many of the RAN selection strategies do consider user requirements and preferences, but in the context of the network-centric vision. These solutions aim to describe the user preferences and then maximise the social welfare, or overall satisfaction, for all the users in a sole operator's network. They use the user preference information to the operator advantage to maximise revenue and network utilisation. For example in [3] Chan et al use their understanding of user behaviour to maximise network gains. They do not consider user-centric approach but rather look at congestion-based pricing to influence the user behaviour with the goal of optimal resource allocation.

A smaller portion of the existing literature consider the always best connected usercentric scenario where users take advantage of the operator competition and select the best network for current service in the current context. Examples are [4] and [5] both of which propose a network selection technique to provide the user with the current best available network, taking into account the user preferences, current available connectivity and the application requirements.

The architecture for implementing the network selection functionality is the focus of much of the work. In designing a new architecture, it is important to limit the modifications required to existing wireless systems, and to minimise the amount of additional network traffic needed. In [6] Eddy addresses the problem of which layer in the IP stack does mobility belong to. He analyses the disadvantages of Mobile IP (a network layer protocol) and concludes that while the Transport Layer is the most likely place for a mobility protocol, a cross-layer approach where interlayer communication is used may be the best approach.

In [7] Stemm et al, an architecture is proposed for vertical handover for multihomed nodes in a wireless overlay network. This is based on Mobile IP but with some modifications. A Handoff Controller is implemented in the mobile terminal to make the decision of which network and which base station (BS) to connect to. This selection decision is based on network availability as indicated by increase or decrease in received beacons from the surrounding BSs, but it may be influenced by network constraints outlined by the user in the User Control Panel (on the terminal) or by heuristic advice from a subnet manager. The focus of their paper is reducing handoff latency; details on the user specified network constraints are not outlined.

Ylitalo et al in [8] look at how to facilitate a user making a network interface selection decision. They concentrate on a possible architecture for the end terminal and do not concentrate on any particular strategy but do mention the Always Cheapest (AC) network selection strategy. Their mechanism is based on Mobile IPv6 with a policy driver module located in the mobile terminal. The user defines their preferences via a GUI in the terminal. These preferences are then interpreted by the policy driver module together with the available information on all the network interfaces to determine a suitable set of actions.

Adamopoulou et al also present a mobile terminal architecture in [4]. They incorporate a GUI on the terminal to collect and weight four parameters (quality, preferred network operator, preferred technology type and cost) in order of the 
indicated user preference for each service in use. Their terminal management architecture consists of a network interface adaptation module, a mobility management module and the user preference module. The network selection is performed by the intelligent access selection function in the mobility management module. The cost information is received from the networks in advance of network selection and is not expected to change frequently. Each network operator charges per data volume, or per unit time, for access at a specific quality level. Multiple simultaneous applications are catered for with different networks selected for each application.

Song et al have their user-centric network selection module implemented in the link layer, with cross-layer signaling messages delivering the QoS information from different layers in the IP-stack, including the application layer where the user describes their desired attributes in a QoS Context. The scheme is based on a large number of parameters which describe; availability, throughput, timeliness, reliability, security, and cost. Two mathematical techniques, analytic hierarchy process and grey relational analysis, are used to perform the analysis and tradeoff between the parameters. The decision maker then chooses the network with the best score.

We focus on the user-centric decision making problem of which available network to choose for data transfer and focus on serving one applications communications needs at a time. The architecture is assumed to be one where the decision-making module is located in the mobile terminal and cross-layer information is available, but the contribution of the paper is the decision process itself and not the architecture.

In [9], H. J. Wang et al describe a handover 'policy' for heterogeneous wireless networks, which is used to select the 'best' available network and time for handover initiation. They consider the cost of using a particular network to be in terms of the sum of weighted functions of bandwidth, power consumption and cost. The network which is consistently calculated to have lowest cost is chosen as the target network. A randomised waiting scheme based on the impact of the estimated handover delay is used to achieve stability and load-balancing in the system, and to avoid handover synchronisation.

A number of papers, all of which reference [9], use similar cost functions. A smart decision model for vertical handoff is the focus of Chen et al in [10]. Their proposed scheme relies on a score function based on functions of allocated bandwidth (transfer completion time), battery power consumption and cost charged by the available networks.

The value or benefit function of offered link capacity is a concave increasing function following the economic assumption of diminishing marginal utility, i.e. value will increase for each unit of added bandwidth up to certain point after which the gain in value for extra capacity is marginal as described as in Murphy et al [11]. The bandwidth in this case is measured using a probing tool. The HTTP handoff decision model presented by Kammann et al in [12] and the work in [13] also use a cost function which is used to compare available networks from the list of options and establish the network to handoff to according to the importance weightings associated with different metrics.

When the wireless terminal seeks information on available access networks, the amount of information supplied should be minimal. Receiving current network condition information may be resource consuming and wasteful in the ever changing 
unreliable wireless environment. We use previous file delays experienced in a particular network to predict transfer completion time for that network.

In [9], bandwidth is determined either by use an agent in each RAN which estimates and broadcasts the current network load, or in the case of commercial networks, by the 'typical' value of bandwidth advertised by these networks. The bandwidth in [10] is measured using a probing tool. The main disadvantage of the probing technique is that it generates traffic adding an overhead in the different RANs. Both [9] and [10] collect current information on bandwidths available on all local networks - this requires heavy power consumption a factor which should possibly be added to the cost of implementing the suggested strategies. Also it is possible that the network becomes more congested just after the information has been collected.

The user network selection decision strategy will be influenced by the pricing scheme employed in the available networks. In our work we assume all available networks employ a price per byte pricing scheme, as i-mode users are currently charged. Our scheme could also cope with other pricing schemes where the cost is known in advance of making the decision, and will not change for the duration of the session.

\section{Consumer Surplus Network Selection Strategy}

In our work we endeavor to find an intelligent solution to the selection decision problem for non real-time data services. In the case of large data transfers, we believe that an initial correct decision may save the user the effort of handoff later in the call session. This decision will rely heavily on the charging schemes implemented by the candidate networks, as users make an effort to get the best value for their money. This paper considers the case when a user wishing to send non real-time data (a large file) has a choice of several available networks, each of which employ a fixed price per byte pricing scheme but each charging different prices. Every user wants timely delivery of their data at the lowest price. While the strict time restrictions imposed by real-time traffic are not used here, it is assumed that every user has a patience limit and will only be willing to wait so long for completed transfer of their data before they become dissatisfied.

We employ a non increasing user utility function to describe user's willingness-topay (in cent) in relation to their willingness-to-wait (in seconds) for a particular service transfer. The more delay a user experiences the less they may be willing to pay. We define two thresholds in terms of time to transfer completion. Any completion time up to $T_{c l}$ is worth the highest price to the user, whereas files arriving after $T_{c 2}$ are worth 0 cent.

In our work in [14], we explored possible shapes for the user utility function. Previous work in the field of economics had related three different utility function shapes with three different users' attitudes to risk taking. The user utility function used in this paper is related to risk neutral user behaviour, where the user equally values paying less money to waiting shorter transfer completion times. It is described in (1) below. 


$$
U_{i}\left(T_{c}\right)= \begin{cases}U_{\max ,} & T_{c} \leq T_{c 1} \\ U_{\mathrm{var}}-T_{c}, & T_{c 1}<T_{c} \leq T_{c 2} \\ 0, & T_{c}>T_{c 2}\end{cases}
$$

The difference between the value of the data to the user (willingness-to-pay) and the actual price they are charged is known in microeconomic terms as the Consumer Surplus (CS). The consumer surplus for sending a file $\mathrm{i}\left(\mathbf{C S}_{\mathbf{i}}\right)$ is described in equation (2) below, where $\mathbf{U}_{\mathbf{i}}\left(\mathbf{T}_{\mathbf{c}}\right)$ is the utility value in cent of transferring file $\mathrm{i}$ in time $\mathbf{T}_{\mathbf{c}}$ seconds and $\mathbf{C}_{\mathbf{i}}$ is the cost charged in cent for sending the file via the chosen network.

$$
\begin{aligned}
& C S_{i}=U_{i}\left(T_{c}\right)-C_{i} \\
& \text { s.t. } T_{c} \leq T_{c 2}
\end{aligned}
$$

In this strategy we use the file size and throughput predictions to predict the transfer completion time. Together with the utility function, this will give us a predicted utility value for each network, which in turn is used together with each network costs to get the predicted consumer surplus. We then choose the network with the best predicted CS.

There are different possible network selection strategies for the user. A user may choose to always stick with one particular network regardless of its current characteristics, for example to always select the cheapest network, or to always chose their preferred operator's network (a network-centric strategy). Another possible strategy would be a random network selection. None of these strategies are responsive to changes in the dynamic radio environment, and in the case that the chosen network is heavily congested the user will be stuck with a poor network choice. However, we believe that an intelligent selection based on user willingness-to-pay, their file completion time constraints and estimated access network delivery time should be used instead. These are considered by our proposed CS-based network selection strategy.

In this paper, a large file is fragmented into smaller chunks and a network selection decision is made for each chunk. The objective of the decision is to transfer the entire file (chunk by chunk) over the current best of the available access networks. It uses the throughput rate information from previous chunks to predict the available rates in each network. We propose a utility-based algorithm that accounts for user time constraints, estimates complete chunk delivery time (for each of the available access networks) and then selects the most promising access network based on consumer surplus difference.

The scenario considered here is where all the network operators employ a fixed pricing scheme. The weighted average of the throughput rates experienced for the last five chunk transactions in a particular network is used as the predicted rate for that network. In the case where there are no previous rates stored for a given network a default rate is used (that is the minimum acceptable rate which will meet the user's 
$T_{c 2}$ ). If the predicted rate for a network is bad then this low rate is only used temporarily before it is deemed stale information and replaced by the default rate.

\section{Access Network Selection Scenario}

An intelligent mobile user's FTP application requires the transfer of a large file (size 100 Kbytes - 5Mbytes) in a SOHWNE. This is a typical range for the size of an FTP transfer. The user terminal has inbuilt intelligent features for network detection and selection. The file is broken into smaller chunks of data (length 10Kbytes). Each data chunk is to be sent uplink from the terminal through the access point (AP) of the selected network to a server in the wired network. The wired network is connected via point-to-point links so that delay in the wired network is negligible and any significant delays experienced are dependent on the selected radio access network. The user may for example be uploading a photo slideshow from a laptop, emailing a large document from their mobile phone, or uploading vending machine data from their PDA to a central server.

The user is faced with a scenario like the one depicted in Figure 1, where the terminal employing the intelligent network selection strategy has a choice of two IEEE 802.11b networks: WLAN0 or WLAN1. We consider that the transfer over WLAN1 is twice as expensive as WLAN0. A repeated decision is required for which RAN to use for transporting each chunk of user's application data.

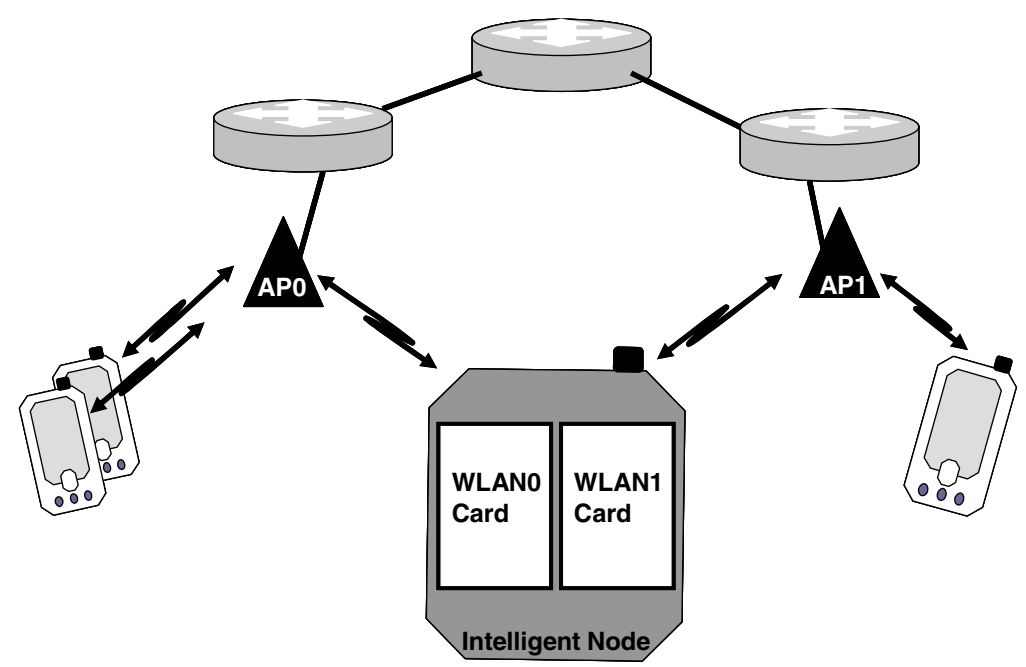

Fig. 1. Simulation scenario setup: user device has a choice of two IEEE 802.11b networks: WLAN0 or WLAN1

The simulation model was developed in NS2 version 2.27 [12] with IEEE 802.11b wireless LAN parameter settings (data rate $11 \mathrm{Mbps}$ ), the NOAH (No Ad-hoc routing) extension, and our new application which simulates a multi-homed terminal 
with a set of inbuilt network selection strategies. Five decision strategies are supported:

- Consumer Surplus Strategy (CS)

- Always Cheapest Strategy (AC)

- Network Centric Strategy (NC)

- Random Strategy (R)

- Another intelligent strategy as proposed in [9] (WKI)

CS network selection strategy conforms to the description presented in section 3 . AS network selection strategy aims at minimizing the cost of data transfer, regardless of the performance of the transmission, selecting the cheapest network available (in this situation WLAN0). NC network selection strategy considers the case when the transfer is always performed over the network owned by the user's preferred network operator (in this case WLAN1 - the more expensive network). The random network selection, randomly selects either WLAN0 or WLAN1. The Wang-Katz I method (WKI) is an implementation of [9] using their formula (3). We implemented the formula with the weightings for cost and bandwidth both set at 0.5 , the weighting for power consumption set to 0 , and used our own throughput prediction scheme. We choose these weightings which convey the situation where the user equally values data delay to the price of the data transfer. The user utility function we used for our CS strategy models the same user behaviour.

$$
\begin{aligned}
& f_{n}=w_{b} \bullet \ln \frac{1}{B_{n}}+w_{p} \bullet \ln P_{n}+w_{c} \bullet \ln C_{n} \\
& \text { s.t. } \sum w_{i}=1
\end{aligned}
$$

The quality of the intelligent node's connectivity in each RAN is affected by the background traffic caused by the other nodes in the cell. In IEEE 802.11b networks if the bulk of data traffic is on the downlink, an FTP session transferring data uplink would have a clear advantage as the bulk of the traffic would create a bottleneck at the AP. The background traffic mix used in this simulation was a mix of HTTP, WAP, FTP and two-way non real time video sessions. The connectivity quality is affected mainly by the background FTP and video traffic. Although WAP is not a common application in current WLANs, in the future when smart-phone users will be taking advantage of the cheaper rates on a WLAN they may be only interested in WAP sessions on their smaller screens.

\section{Comparison-Based Performance Evaluation}

During successive tests file size is varied from $100 \mathrm{Kbytes}$ to 5 Mbytes. All five network selection strategies: CS, AC, NC, R and WKI were employed in turn. In the case of CS network selection strategy the intelligent node will shortlist the networks based on delivery time prediction to meet $T_{c 2}$. Each chunk is then transmitted over the 
short-listed RAN which is predicted to maximise the CS. If none of the available networks can meet the delivery deadline, then the cheapest network is selected.

If a chunk transfer exceeds the transfer completion deadline, the transmission is stopped and the chunk is resent. The entire file transfer completion time, cost of transfer, number of chunk resends required and consumer surplus are then compared against two other network selection strategies: AC and NC. The background traffic pattern on WLAN0 changes from high traffic to medium whereas WLAN1 starts with a medium amount of traffic which increases. As WLAN1 is twice as expensive as WLAN0 we assume that it will get slightly less traffic and allowed for that in the background traffic patterns.

In the experiments, it was noted that the WKI strategy only ever selected the cheapest network, WLAN0. This was due to the default setting for our throughput prediction scheme. As WLAN1 was twice as expensive as WLAN0, it needed to be twice as fast in order to be selected under the WKI scheme. With our default throughput setting, which was low, WKI strategy never selected WLAN1 and so the actual throughput rates available on WLAN1 were not identified. As a result of this, we changed the default setting to a higher bandwidth and re-ran tests for 5Mbyte files under the strategy name WKII. While WKII compares favourably with our strategy on the average transfer completion time, it does not fair as well on the chunk resends and the average cost per file.

\subsection{Average Delay Per File Transfer}

The average transfer completion delay per file (measured in seconds) is compared for the all the network selection strategies and the results are plotted in figure 2 . The

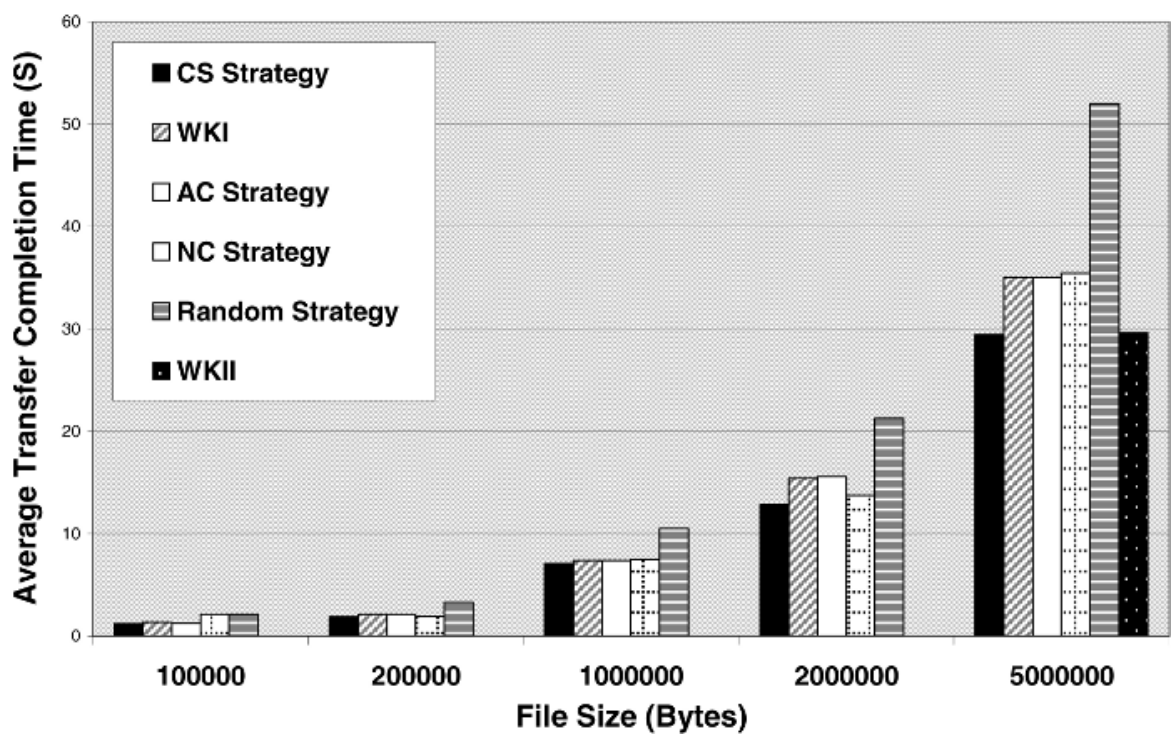

Fig. 2. Average File Transfer Completion Time 
transfer completion time is important to the non real-time users as they are not willing to wait too long for the file transfer to finish. The sooner the file transfer ends, the more satisfied the user is. Analyzing the results it can be seen that the average duration for files sent with the CS-based strategy are the shorter than files sent with any of the other methods, with the exception of WKII. As file size increases the difference between the CS average transfer time and the others increases. For example when transferring 5000000 byte files using CS strategy, 17\% shorter times for transfers are recorded than when AC strategy is employed and 19\% shorter than when $\mathrm{NC}$ approach is used.

\subsection{Average Cost Per File Transfer}

The user wants to take advantage of the competition and pay as little as possible to achieve the expected perceived quality for the current service. Figure 3 shows the comparison of average cost (in cent) per file transfer using each of the three strategies considered in this paper. As expected with the NC strategy, users who always choose their network operator's WLAN (WLAN1) end up paying the most as this is the most expensive network of the two. For the case of transferring the maximum file size considered in this paper, users on the NC scheme pay on average double what users on the AC scheme pay. Although as expected the AC strategy achieves the least costly transfers, the difference between the average costs paid per file for the CS and AC strategy users is not very much, compared to the difference in average delay.

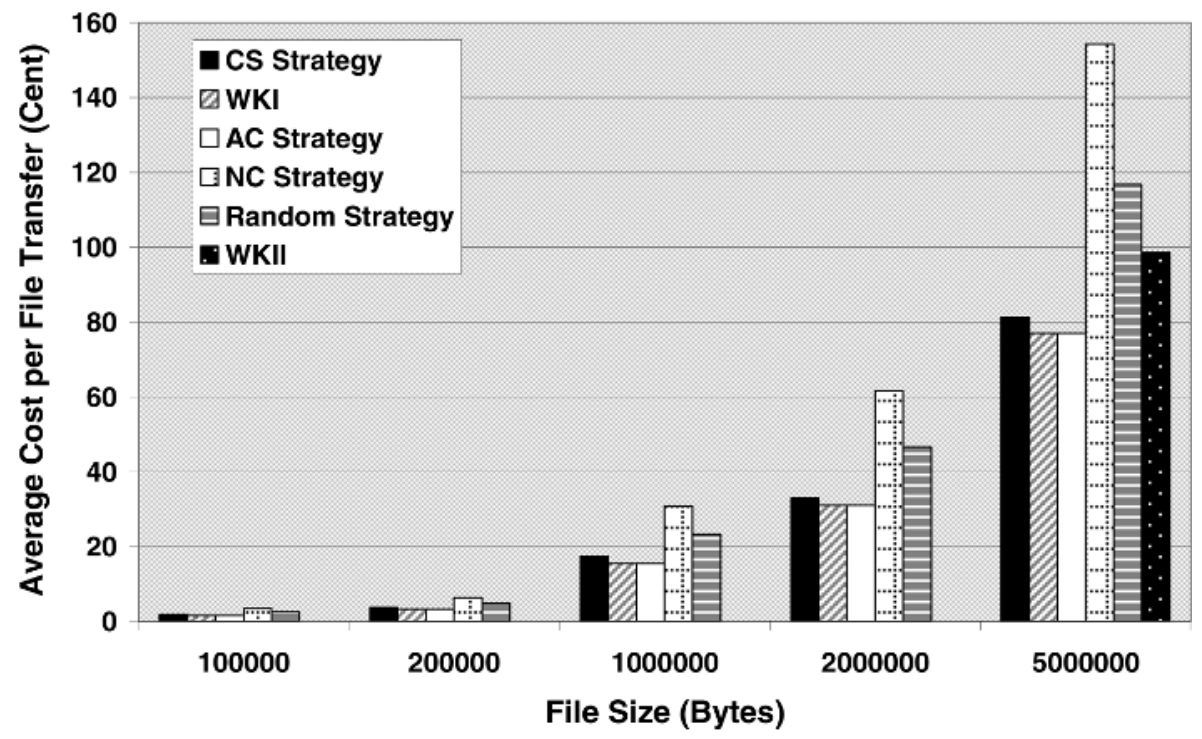

Fig. 3. Average Cost per File Transfer 


\subsection{Transmission Efficiency Per File Transfer}

One measurement of transmission efficiency is in terms of how many network resources were used in order to transfer a given amount of data. Looking at the

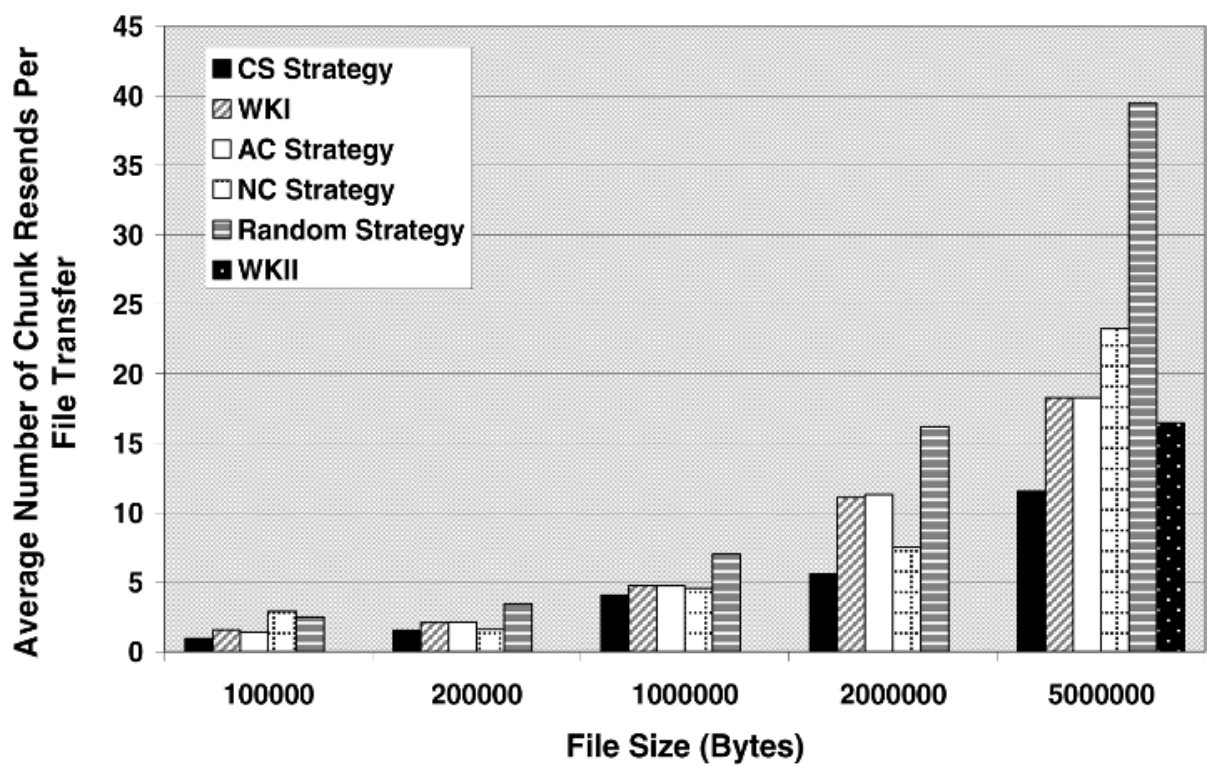

Fig. 4. Average Number of Chunk Resends per File Transfer

number of chunk resends counted when each of the network selection strategies were employed presented in figure 4 it is obvious that while customers employing the AC or WKI strategy are paying less per chunk sent, their chunks exceed the time deadline more often and have to be resent, costing these customers more in time, money and battery consumption in the long run. As file size increases the advantage of using the CS strategy becomes more apparent, chunk resends are far less than those recorded in all the other cases. It is significant to highlight the gains of $39 \%$ in comparison with the AC strategy and $52 \%$ in comparison with the NC plan obtained on average in chunk resends during the 5000000 byte file transfers.

\subsection{Summary}

Table 1 summarizes the results of using different strategies for the transfer of files with various sizes. 
Table 1. Comparison between CS, AC and NC strategies when transferring different size files

\begin{tabular}{lcccc}
\hline File Size & Strategy & $\begin{array}{c}\text { Average } \\
\text { Duration } \\
\text { (seconds) }\end{array}$ & $\begin{array}{c}\text { Average } \\
\text { Cost } \\
\text { (cents) }\end{array}$ & $\begin{array}{c}\text { Average } \\
\text { Resends } \\
\text { (times) }\end{array}$ \\
\hline 100000 & CS & 1.184 & 1.782 & 0.980 \\
& AC & 1.233 & 1.609 & 1.417 \\
& NC & 2.133 & 3.419 & 2.916 \\
\hline 200000 & CS & 1.882 & 3.661 & 1.528 \\
& AC & 2.130 & 3.142 & 2.117 \\
& NC & 1.925 & 6.275 & 1.610 \\
\hline 1000000 & CS & 7.045 & 17.357 & 4.072 \\
& AC & 7.337 & 15.465 & 4.763 \\
& NC & 7.486 & 30.822 & 4.580 \\
\hline 2000000 & CS & 12.833 & 32.992 & 5.604 \\
& AC & 15.551 & 31.035 & 11.327 \\
& NC & 13.746 & 61.527 & 7.529 \\
\hline 5000000 & CS & 29.444 & $\mathbf{8 1 . 3 0 4}$ & 11.556 \\
& AC & 35.040 & 76.938 & 18.280 \\
& NC & 35.406 & 154.152 & 23.250 \\
& WKII & 29.579 & $\mathbf{9 8 . 5 2 2}$ & 16.464 \\
\hline
\end{tabular}

\section{Conclusions and Future Work}

Thanks to deregulation and the advancements in technologies, the user is becoming more powerful in their everyday consumer choice. Users in a user-centric SOHWNE will be free to choose the available access network which can best satisfy their needs in their current situation. This paper proposes, describes and evaluates a novel algorithm for intelligent cost-oriented and performance-aware selection between available networks. This utility-based model for user network selection divides large files into smaller chunks and selects the best available network for transferring each chunk, with user-specified time constraints, in a heterogeneous wireless environment.

The ultimate goal is to find the best user-centric network selection strategy for non real-time data transfer in the next generation service oriented heterogeneous wireless network environment. In this paper the proposed Consumer Surplus (CS)-based strategy was evaluated against an Always Cheapest (AC), a Network Centric (NC), a Random and two forms of an intelligent selection strategy (WKI \& WKII). Test results showed that of all the comparison strategies WKII was the strongest contender.

WKII compares favourably with our CS strategy on the average transfer completion time, but it is not quite good as on the chunk resends and the average cost per file transfer.

More rigorous testing of the CS in compassion with forms of the WK strategy are currently underway, including scenarios with different competing traffic mixes and different chunk sizes.

Future models will use more advanced throughput prediction methods that consider the time of day, day of week, previous history, congestion recovery time and other possible influential metrics. 
Acknowledgments. The support of the Informatics Commercialisation initiative of Enterprise Ireland is gratefully acknowledged.

\section{References}

1. Houssos, N., Gazis, V., Panagiotakis, S., Gessler, S., Schuelke, A., Quesnel, S.: Value Added Service Management in 3G Networks. IEEE/IFIP Network Operations and Management Symposium (2002)

2. Kanter, T.: Going Wireless: Enabling an Adaptive and Extensible Environment. ACM Journal on Mobile Networks and Applications, Vol. 8, No. 1 (2003) 37-50

3. Chan, H., Fan, P., Cao, Z.: A Utility-Based Network Selection Scheme for Multiple Services in Heterogeneous Networks. IEEE International Conference on Wireless Networks, Communications and Mobile Computing (2005)

4. Adamopoulou, E., Demestichas, K., Koutsorodi, A., Theologou, M.: Intelligent Access Network Selection in Heterogeneous Networks - Simulation Results. IEEE Wireless Communication Systems Symposium (2005) 279-283

5. Song, Q., Jamalipour, A.: An adaptive quality-of-service network selection mechanism for heterogeneous mobile networks. IEEE Wireless Communications and Mobile Computing (2005) 697-708

6. Eddy, W. M.: At What Layer Does Mobility Belong? IEEE Communications Magazine (2004)

7. Stemm M., Katz, R.H.: Vertical handoffs in wireless overlay networks. Mobile Networks and Applications 3 (1998)

8. Ylitalo, J., Jokikyyny, T., Kauppinen, T., Tuominen, A. J., Laine, J.: Dynamic Network Interface Selection in Multihomed Mobile Hosts. $36^{\text {th }}$ Hawaii International Conference on System Sciences (2003)

9. Wang, H.J., Katz, R.H., Giese, J.: Policy-enabled handoffs across heterogeneous wireless networks. Second IEEE Workshop on Mobile Computing Systems and Applications (1999)

10. Chen, L-J., Sun, T., Chen, B., Rajendran, V., Gerla, M.: A Smart Decision Model for Vertical Handoff. 4th ANWIRE International Workshop on Wireless Internet and Reconfigurability (2004)

11. Murphy, J., Murphy, L.: Bandwidth Allocation By Pricing In ATM Networks. IFIP Transactions, C-24, pp. 333-351, Broadband Communications II (1994)

12. Angermann, M., Kammann, J.: Cost Metrics For Decision Problem In Wireless Ad Hoc Networking. IEEE CAS Workshop on Wireless Communications and Networking (2002)

13. McNair, J., Zhu, F.: Vertical Handoffs in Fourth-Generation Multinetwork Environments. IEEE Wireless Communications (2004) 8-15

14. Ormond, O., Muntean, G.-M., Murphy, J.: Utility-based Intelligent Network Selection in Beyond 3G Systems. IEEE International Conference on Communications (2006)

15. Network Simulator-2, [Online]. Available on http://www.isi.edu/nsnam/ns/ 